\title{
EL DESCENSO DE LAS ACTITUDES HACIA LA CIENCIA DE CHICOS Y CHICAS EN LA EDUCACIÓN OBLIGATORIA
}

\section{The decline of girls' and boys' attitudes toward science in compulsory education}

\author{
Ângel Vázquez-Alonso ${ }^{1}$ \\ María Antonia Manassero-Mas²
}

\begin{abstract}
Resumen: Este estudio analiza empíricamente las actitudes relacionadas con la ciencia a través de tres dimensiones (imagen de la ciencia y la tecnología, ciencia escolar y preservación del medio ambiente), por sexo y edad, en una muestra de estudiantes españoles desde cuarto grado en adelante. La evolución de las actitudes hacia la ciencia escolar con el tiempo exhiben un perfil común de fuerte descenso global (son más positivas en los primeros grados de la escuela y van disminuyendo al aumentar la edad). Las actitudes hacia la ciencia escolar exhiben un gran descenso con la edad, mientras la imagen de la ciencia o el medio ambiente no están tan deterioradas; según el sexo, chicos y chicas exhiben patrones de descenso diferenciados. Se discuten algunas implicaciones de los resultados para la educación científica en la escuela y las vocaciones científicas, así como las limitaciones del estudio.

Palabras clave: Educación científica. Actitudes relacionadas con la ciencia. Evolución longitudinal. Diferencias por sexo.
\end{abstract}

\begin{abstract}
An empirical analysis of the deterioration of the science related attitudes across students' age and sex is presented. The attitudinal factors embrace a group of variables on the image of science, environmental preservation and some aspects of school science and have been analyzed in a sample of students across several ages starting at the fourth grade. The decline mainly affects the attitudes toward school science, while the attitudes towards the image of science or the environmental preservation do not exhibit this deterioration. The time evolution of the students' answers demonstrates the global decrease of attitudes across age and gender: the first grades display the best attitudes, then they significantly decrease as the age increase; moreover boys and girls display different profiles of attitudinal decay across time. The implications of these results for school science education and the limitations of the study are discussed.
\end{abstract}

Keywords: Science education. Attitudes related to science. Evolution across age. Gender differences.

${ }^{1}$ Licenciado en Ciencias Físicas y Ciencias Químicas, doctor en Ciencias de la Educación. Profesor, Departamento de Pedagogía Aplicada y Psicología de la Educación, Universidad de las Islas Baleares. Islas Baleares. Palma de Mallorca, Islas Baleares, España.<angel.vazquez@uib.es>

${ }^{2}$ Licenciada y doctora en Psicología. Catedrática de Psicología Social, Departamento de Psicología.

<ma.manassero@uib.es>

${ }^{1}$ Carretera de Valldemossa, $\mathrm{km} 7.5$ - 07071,

Palma de Mallorca, España 
Vázques-Alonso, Â.; Manassero-Mas M. A.

\section{Introducción}

Numerosos estudios y revisiones en las últimas décadas han informado acerca de las negativas e inadecuadas actitudes de los estudiantes hacia la ciencia, y más específicamente, la falta de interés hacia la ciencia en la escuela (GARDNER, 1975; HAVARD, 1996; JENKINS; NELSON, 2005; ORMEROD; DUCKWORTH, 1975; OSBORNE; SIMON; COLLINS, 2003; PIBURN; BAKER, 1993; SCHIBECI, 1984; VÁZQUEZ ALONSO; MANASSERO MAS, 1995; WEINBURGH, 1995). La importancia de esta cuestión radica en la reconocida influencia de las actitudes sobre las conductas de aprendizaje escolar de la ciencia, que se proyectan a través de variables intermedias como motivación, sentido de autoeficacia, objetivos a largo plazo, etc.; las actitudes positivas favorecen el aprendizaje y el rendimiento, mientras las actitudes negativas los interfieren y disminuyen (AJZEN; FISHBEIN, 1980; EAGLY; CHAIKEN, 1993; FAZIO, 1986). Hasta tal punto son importantes las actitudes que para algún especialista (FENSHAM, 2004), las actitudes negativas de los estudiantes constituyen el principal problema que deben afrontar actualmente la educación científica y la didáctica de la ciencia; por otro lado, este consenso sobre la importancia práctica de las actitudes converge con un indicador reciente y bien significativo: en la última evaluación centrada en ciencias el programa internacional PISA incluye las actitudes de los estudiantes hacia la ciencia como un objetivo central y encuentra que los estudiantes están dispuestos a proseguir una carrera científica si valoran positivamente la ciencia o disfrutan con el estudio de la ciencia (ORGANISATION FOR ECONOMIC CO-OPERATION AND DEVELOPMENT, 2007).

Las actitudes relacionadas con la ciencia dependen de diversos factores como han puesto de manifiesto numerosas investigaciones (MANASSERO-MAS; VÁZQUEZ ALONSO, 1996; SIMPSON; OLIVER, 1990), entre los cuales el sexo de los estudiantes es uno de los más importantes (FENSHAM, 2004; GREENFIELD, 1996; HENDLEY et al., 1995; JIMÉNEZ; ÁLVAREZ, 1992; LEE; BRYK, 1986). En general, las actitudes relacionadas con la ciencia de las mujeres son peores que las de los hombres, aunque el tamaño de las diferencias depende, a su vez, de factores muy diversos, relacionados con la educación, la cultura o el país (SJØBERG, 2005; TRUMPER, 2004). En el mencionado estudio de PISA 2006 (ORGANISATION FOR ECONOMIC CO-OPERATION AND DEVELOPMENT, 2007) muchos países exhiben las clásicas diferencias favorables a los hombres, aunque las diferencias de género en rendimiento general ya no sean significativas en muchos países (caso de España).

Algunos trabajos refieren que el cambio negativo de las actitudes se produce al inicio de la adolescencia, aproximadamente en torno a los 12 años, momento que se corresponde también con la transición entre la etapa de primaria y la secundaria; entonces, la curiosidad e interés naturales de los niños hacia la ciencia comienzan a transformarse en desinterés, aburrimiento y experiencias de fracaso escolar, y esta depresión es más intensa para las mujeres (GEORGE, 2000; GIBSON; CHASE, 2002; MURPHY; BEGGS, 2003; PELL; JARVIS, 2001; PIBURN; BAKER, 1993; RAMSDEN, 1998; SIMPSON; OLIVER, 1990).

Esta preocupante y progresiva depresión actitudinal hacia la ciencia se atribuye a que la ciencia escolar se va ganando una creciente imagen negativa (autoritaria, aburrida, difícil, irrelevante para la vida diaria y causa de los problemas medio-ambientales que preocupan a la opinión pública) en la mente de los estudiantes. Esta progresiva falta de interés va alejando a los adolescentes de la ciencia escolar, de modo que la consecuencia natural es el abandono de 
El descenso de las actitudes hacia la ciencia ...

la ciencia y las carreras científicas por los jóvenes en las primeras elecciones de estudios y carreras, un resultado que preocupa actualmente porque causa un descenso de las vocaciones científicas, un problema acuciante para los países europeos (CONSEJO DE EUROPA, 2003; GAGO, 2004; ROCARD, 2007).

Algunos estudios sostienen que en la etapa de primaria los estudiantes tienen bastante entusiasmo e interés por las actividades de aprendizaje en la clase de ciencias, y también que este interés es más bajo en la etapa de educación secundaria, y especialmente en algunas materias, como física y química (OSBORNE; DRIVER; SIMON, 1998; PARKINSON et al. 1998; RAMSDEN, 1998; SIMPSON; OLIVER, 1990; WEINBURG, 1995). Sin embargo otros estudios sugieren que esta erosión de las actitudes hacia la ciencia, admitida y confirmada en secundaria, incluso podría empezar al final de la educación primaria (PELL; JARVIS, 2001; MURPHY; BEGGS, 2003).

La explosión hormonal característica de la adolescencia provoca drásticos cambios afectivos que justifican y fundamentan estos cambios observables en las actitudes, la personalidad y la conducta de los adolescentes. El diferente desarrollo evolutivo entre chicos y chicas (en general, las chicas maduran emocionalmente antes, y de manera diferente que los chicos), concomitante con esos radicales cambios afectivos propios de la adolescencia, genera profundas diferencias en actitudes, ritmos, estilos y formas de conductas. La reconocida marca de género masculina atribuida a la ciencia y la tecnología (en adelante $\mathrm{Cy} T$ ) estereotipa ambas como disciplinas y profesiones más propias de hombres que de mujeres (p.e. las mujeres son minoría en $\mathrm{Cy} T$ ), de modo que atraen más a los hombres que a las mujeres (VÁZQUEZ; MANASSERO, 2007a, 2009, 2010). Este estereotipo puede estar en la base del decrecimiento del interés de los jóvenes hacia la CyT que ocurre en la adolescencia, y además con un perfil marcadamente diferenciado para chicos y chicas: los chicos mantienen una actitud más positiva y mejor rendimiento en ciencias que las chicas, mientras estas rechazan crecientemente la $\mathrm{Cy} \mathrm{T}$ y obtienen peor rendimiento (a pesar de que las chicas superan ampliamente a los chicos en el rendimiento escolar de otras materias, especialmente el lenguaje). Otros estudios aportan matices específicos sobre este patrón general de las diferencias de género, pues atribuyen a las chicas actitudes hacia la ciencia más positivas que los chicos en primaria y al comienzo de la educación secundaria (MURPHY; BEGGS, 2003); otros sugieren que el descenso de las actitudes de las chicas en secundaria podría ser mayor que el de los chicos, especialmente en física (BAIRD; PENNA, 1997; FERGUSON; FRASER, 1996; SPEERING; RENNIE, 1996).

Las actitudes hacia CyT dependen también de la educación, la cultura y el país. La ciencia está entre las asignaturas escolares más difíciles y que menos gustan en diferentes países anglosajones (HENDLEY et al., 1995; HENDLEY; STABLES; STABLES, 1996). Este rechazo hacia la ciencia parece más alto en otros países occidentales que en España (INSTITUTO NACIONAL DE EVALUACIÓN Y CALIDAD DEL SISTEMA EDUCATIVO, 2003; MONGUILLOT, 2002; PÉREZ, 2005), donde las asignaturas de ciencias no son de las que menos gustan a los estudiantes españoles, aunque su interés por ellas, ciertamente, decrece con el tiempo; al final de primaria e inicio de secundaria la ciencia no está entre las asignaturas menos preferidas, y al final de secundaria, a pesar del decrecimiento del interés en todas las asignaturas, la ciencia se encuentra aún entre las más interesantes.

El análisis comparativo entre más de 40 países participantes en el estudio ROSE (SCHREINER; SJØBERG, 2004) demuestra que las actitudes relacionadas con la ciencia y la 
tecnología en estudiantes de final de secundaria ofrecen un patrón general que resulta inversamente proporcional al grado de desarrollo: los estudiantes de los países occidentales más desarrollados (que obtienen un mejor rendimiento en las pruebas internacionales de aprendizaje y conocimientos) tienen actitudes generales hacia la ciencia más negativas que los estudiantes homólogos de los países en desarrollo (SJØBERG, 2004). Estos resultados también sugieren la influencia de factores culturales y sociales, que actuarían como variables moduladoras de las actitudes hacia la ciencia escolar (BREAKWELL; BEARDSELL, 1992; SJØBERG, 2000).

En suma, aunque algunos estudios reiteran la existencia de un deterioro de las actitudes hacia la ciencia a lo largo de la escolarización, pocos estudios han afrontado empírica y directamente esta cuestión y aún menos sus diferencias de género. Este trabajo presenta un análisis empírico de este tema en una muestra de estudiantes españoles de primaria y secundaria, para validar empíricamente la hipótesis siguiente: el descenso de las actitudes hacia la ciencia que tiene lugar desde la educación primaria hasta los últimos años de la educación secundaria tiene perfiles diferenciados para hombres y mujeres.

\section{Metodología}

\section{Muestra}

Este estudio emplea una muestra opinática, jóvenes visitantes en la Feria de la Ciencia 2007 de las Islas Baleares. La gran mayoría de los cuestionarios fueron realizados por clases completas de estudiantes bajo la supervisión de sus profesores, que decidieron anónima y voluntariamente contestar el cuestionario de actitudes hacia la ciencia, como una actividad complementaria de la Feria y que es la base de este estudio (tabla 1). Esta circunstancia confiere a la muestra un cierto equilibrio entre chicos y chicas, al provenir mayoritariamente de las mismas clases.

La muestra final está formada por 678 personas que respondieron válidamente el cuestionario, después de depurar y eliminar algunos casos (cuestionarios muy incompletos o deficientemente cumplimentados, etc.). La edad de los participantes se extiende desde los 9 a los 59 años, pero casi exclusivamente (96\%) corresponde a estudiantes entre 9 y 17 años.

Un poco más de la mitad de la muestra son chicos (361, 53\%) y el resto chicas (326, $47 \%$ ). Por etapas educativas, los participantes se distribuyen entre la educación primaria (32\%), secundaria obligatoria $(60 \%)$, post-secundaria (bachillerato -4\%- y formación profesional $1,7 \%-)$, universidad $(0,4 \%)$ y sin estudios $(1,2 \%)$. Por cursos, cabe destacar que los estudiantes de primaria corresponden sólo a los tres últimos cursos de la etapa $\left(4^{\circ}, 5^{\circ}\right.$ y $\left.6^{\circ}\right)$.

La elección de asignaturas de ciencias en el sistema educativo es realizada por vez primera en el grado 10 (se puede elegir Física y Química y/o Biología y Geología frente a otras asignaturas). Puesto que la gran mayoría de la muestra se encuentra en estudios donde la ciencia es una asignatura obligatoria y sólo una muy pequeña minoría $(2 \%)$ cursan ciencias en estudios donde éstas ya no son obligatorias, y por tanto son elegibles, esta variable no se considera en los análisis. 
El descenso de las actitudes hacia la ciencia ...

Tabla 1. Tabulación cruzada de la muestra válida por estudios, sexo y curso (se omiten los casos que tienen valores perdidos en alguna de las tres variables).

\begin{tabular}{|c|c|c|c|c|c|c|c|c|c|c|c|}
\hline \multirow{3}{*}{\multicolumn{2}{|c|}{ Estudios }} & \multirow{3}{*}{ Sexo } & \multicolumn{8}{|c|}{ Grados } & \multirow{3}{*}{ Total } \\
\hline & & & \multicolumn{3}{|c|}{ Primaria } & \multicolumn{4}{|c|}{ Secundaria obligatoria } & \multirow[b]{2}{*}{ Otros } & \\
\hline & & & 4 & 5 & 6 & 7 & 8 & 9 & 10 & & \\
\hline \multicolumn{12}{|c|}{ Primaria (9 - 12 años) } \\
\hline & & Hombres & 48 & 59 & 12 & & & & & & 119 \\
\hline & & Mujeres & 41 & 45 & 16 & & & & & & 102 \\
\hline & Total & & 89 & 104 & 28 & & & & & & 221 \\
\hline \multicolumn{12}{|c|}{ Secundaria (13 -16 años) } \\
\hline & & Hombres & & & & 51 & 79 & 59 & 25 & & 214 \\
\hline & & Mujeres & & & & 55 & 65 & 53 & 28 & & 201 \\
\hline & Total & & & & & 106 & 144 & 112 & 53 & & 415 \\
\hline \multicolumn{12}{|c|}{ Bachillerato (17-18 años) } \\
\hline & & Hombres & & & & & & & & 15 & 15 \\
\hline & & Mujeres & & & & & & & & 13 & 13 \\
\hline & Total & & & & & & & & & 28 & 28 \\
\hline \multicolumn{12}{|c|}{ Formación profesional (17-18) } \\
\hline & & Hombres & & & & & & & 8 & 3 & 11 \\
\hline & Total & & & & & & & & 8 & 3 & 11 \\
\hline \multicolumn{12}{|l|}{ Universidad } \\
\hline & & Hombres & & & & & & & & & 1 \\
\hline & & Mujeres & & & & & & & & & 1 \\
\hline & Total & & & & & & & & & & 2 \\
\hline No estudiantes & & Mujeres & & & & & & & & & 1 \\
\hline & Total & & & & & & & & & & 1 \\
\hline
\end{tabular}

Casos perdidos: estudios (3), sexo (6), curso (11), edad (3).

\section{Instrumento}

El instrumento de la investigación es un cuestionario de actitudes hacia la ciencia elaborado específicamente para este estudio, formado por 24 cuestiones que contienen valoraciones de diversos rasgos acerca de la ciencia, la ciencia escolar y el medio ambiente cuyo texto se ofrece en el apéndice. Cada cuestión es una frase breve que los estudiantes valoran anónimamente sobre una escala Likert de cuatro puntos (1 a 4) para expresar su grado de acuerdo o desacuerdo con ella.

Este cuestionario ha sido construido con diversos ítems significativos del instrumento utilizado en el proyecto ROSE (SCHREINER; SJØBERG, 2004), cuya aplicación y análisis empíricos completos se desarrollaron e informaron en estudios previos (VÁZQUEZ ALONSO; MANASSERO MAS, 2007a). El contenido de las cuestiones seleccionadas se centra en tres escalas del proyecto ROSE: 7 cuestiones de la escala imagen de la ciencia (denotadas con IM), 15 cuestiones de la escala de actitudes hacia la ciencia escolar (denotadas CE) y 2 cuestiones de la escala de actitudes hacia el medio ambiente (denotadas MA). La fiabilidad empírica del cuestionario es alta (alfa de Cronbach $=0,8878$ ).

Las cuestiones de actitudes hacia la ciencia escolar son las más numerosas y para facilitar el análisis de este estudio se dividen en tres grupos (básicas, vocacionales y objetivos), conforme a los resultados obtenidos de un análisis previo de la estructura factorial y cuya 
asignación figura en las tablas (VÁZQUEZ ALONSO; MANASSERO MAS, 2007a). Las variables de actitudes básicas comprenden aquellas que indican las disposiciones más generales hacia la ciencia escolar como el interés (\#8), la facilidad de su aprendizaje (\#9), el agrado (\#12) y el aprendizaje de ciencia para todos (\#13); las variables vocacionales son rasgos indicadores de disposiciones vocacionales hacia la CyT como apertura a nuevos trabajos (\#10), utilidad para el trabajo (\#11), mejora de oportunidades (\#15), deseo de ser científico (\#23) y deseo de trabajar en tecnología (\#24); las variables de objetivos son aquellas que recogen la percepción de los estudiantes hacia aspectos que constituyen objetivos generales de la ciencia escolar, tales como la utilidad para la vida cotidiana (\#14), desarrollar la capacidad de crítica y escepticismo (\#16), aumentar la curiosidad (\#17), cuidado de la salud (\#18), importancia de la ciencia (\#19) y aprecio por la naturaleza (\#20).

Además de los contenidos centrales de la encuesta, los participantes respondieron (anónimamente) también algunos datos sociodemográficos personales, tales como el sexo, la edad, los estudios y el curso actuales y si ya estudian asignaturas optativas de ciencias.

\section{Procedimiento}

El cuestionario del estudio se puso a disposición de los participantes y visitantes de la Feria de la Ciencia 2007 de las Islas Baleares de dos formas diferentes: en formato papel, en un punto del recinto de la Feria, y en formato electrónico en la página web oficial de la Feria. La mayoría de los cuestionarios (546) fueron contestados en papel, y de ellos, la mayor parte fueron aplicados por profesores en sus aulas como una actividad de aula previa a la visita del grupo a la Feria. Como variables dependientes se consideran las puntuaciones actitudinales de las diversas cuestiones del instrumento y como variables independientes, las variables sociodemográficas (sexo, edad, estudios, curso, etc.). Para contrastar la hipótesis de la disminución con el tiempo de las actitudes hacia la CyT de los estudiantes, se comparan las actitudes entre las etapas de primaria y secundaria y a lo largo de los diferentes grados; se excluyen los estudiantes de cursos superiores a tercero de ESO, porque en su mayoría son estudiantes que han elegido ciencias y podrían sesgar el resultado.

La hipótesis a verificar es el declive de las actitudes en chicos y chicas a medida que progresan por los sucesivos grados escolares, así como las diferencias entre ambos grupos en esta evolución. Como criterios de verificación se consideran la significación de las diferencias y la evolución del declive; se considera verificada la hipótesis cuando se observen diferencias estadísticamente significativas entre los grados y un decrecimiento desde los primeros a los últimos grados.

Los distintos subgrupos de etapas y grados se comparan mediante análisis de la varianza (criterio de significación estadística p < .01) para los que se indica el grado de significación de las diferencias y el tamaño del efecto observado (diferencia entre las medias de los grupos medida en unidades de desviación estándar promedio). Este último estadístico permite valorar la relevancia de la magnitud de las diferencias encontradas; tamaños $<.30$ suelen considerarse irrelevantes, aunque algunos tamaños irrelevantes pueden ser estadísticamente significativos (para $\mathrm{p}<.01$ ).

Para simplificar el análisis y la descripción de los perfiles de descenso actitudinal se usan las siguientes categorías: 
El descenso de las actitudes hacia la ciencia ...

- Descenso regular: la puntuación desciende continuamente del primero al último grado.

. Descenso abrupto en primaria: la mayor proporción del descenso observado ocurre en los tres primeros grados (primaria), y es casi constante en los tres siguientes grados de secundaria.

. Descenso abrupto en secundaria: la mayor proporción del descenso observado ocurre en los tres últimos grados (secundaria), y es casi constante en los tres primeros grados de primaria.

. Descenso irregular: la tendencia general de descenso presenta en algunos repuntes al alza en grados intermedios (aspecto en dientes de sierra).

\section{Resultados}

Las valoraciones globales de las distintas cuestiones para toda la muestra son generalmente positivas, pues en la casi totalidad de las cuestiones la puntuación media se encuentra por encima del punto central de la escala empleada ( 2,5 puntos) que, por ello, podría considerarse la frontera entre actitudes positivas y negativas (tabla 2).

El ANOVA comparativo global entre chicas y chicos sobre toda la muestra indica que las diferencias estadísticamente significativas son realmente escasas, pues sólo dos cuestiones producen diferencias estadísticamente significativas, y ambas favorables a los chicos: la ciencia escolar me gusta más que la mayoría de las otras asignaturas - CEgust12 - $(\mathrm{F}(1,676)=9,00$; $\mathrm{p}$ $<0,003)$ y me gustaría conseguir un trabajo en tecnología - CEtrtg24 $-(\mathrm{F}(1,672)=53,86 ; \mathrm{p}=$ 6E-13). En ambas cuestiones, los chicos tienen puntuaciones significativamente mayores que las chicas, pero como en el resto de las cuestiones, las diferencias entre chicos y chicas no son estadísticamente significativas (aunque los chicos alcanzan puntuaciones superiores a las chicas en la gran mayoría), no cabría concluir que las diferencias de género en las actitudes hacia la ciencia sean importantes.

$\mathrm{El}$ análisis de las variables actitudinales que diferencian significativamente a chicos y chicas en función del tamaño del efecto permite valorar su importancia efectiva. La primera indica que la ciencia escolar gusta significativamente más a los chicos que a las chicas, aunque como el tamaño del efecto de las diferencias es aún pequeño $(-0,232)$ y no se puede considerar relevante. En el caso de los deseos de conseguir un trabajo en tecnología, la diferencia es ya moderadamente grande (tamaño -0,566) pues alcanza un valor superior a la mitad de una desviación estándar. Esta última cuestión marca una diferenciación notable en la inclinación vocacional de chicas y chicos, no sólo cuantitativa, como indica el gran tamaño de la diferencia, sino también cualitativa, pues mientras los chicos valoran positivamente un trabajo en tecnología, las chicas lo valoran negativamente (por debajo el punto medio de la escala de valoración).

En suma, los chicos tienen actitudes globales más positivas que las chicas en la mayoría de las variables evaluadas, pero las diferencias no son estadísticamente significativas excepto en dos casos, favorables ambos a los chicos: el gusto por la ciencia escolar y el deseo de tener un trabajo en tecnología. Sin embargo, las diferencias son importantes sólo en el segundo de ellos. 
Vázques-Alonso, Â.; Manassero-Mas M. A.

Tabla 2. Puntuaciones medias de las cuestiones totales y por sexos.

\begin{tabular}{|c|c|c|c|c|c|c|c|c|c|c|}
\hline \multirow[b]{2}{*}{ Cuestión } & \multicolumn{3}{|c|}{ Hombres } & \multicolumn{3}{|c|}{ Mujeres } & \multicolumn{3}{|c|}{ Total } & \multirow{2}{*}{$\begin{array}{c}\text { Tamaño } \\
\text { del } \\
\text { efecto }\end{array}$} \\
\hline & Media & Desv. est. & $\mathbf{N}$ & Media & Desv. est. & $\mathbf{N}$ & Media & Desv. est. & $\mathbf{N}$ & \\
\hline IMcurar1 & 3,44 & 0,75 & 355 & 3,31 & 0,82 & 321 & 3,38 & 0,79 & 676 & $-0,155$ \\
\hline IMoportu2 & 3,41 & 0,74 & 355 & 3,33 & 0,80 & 323 & 3,37 & 0,77 & 678 & $-0,104$ \\
\hline IMvida3 & 3,26 & 0,81 & 355 & 3,17 & 0,75 & 322 & 3,22 & 0,78 & 677 & $-0,124$ \\
\hline IMtrabaj4 & 2,99 & 0,83 & 351 & 2,95 & 0,81 & 320 & 2,97 & 0,82 & 671 & $-0,050$ \\
\hline IMbalan5 & 2,83 & 0,88 & 337 & 2,74 & 0,85 & 304 & 2,79 & 0,87 & 641 & $-0,098$ \\
\hline IMimpor6 & 3,30 & 0,76 & 345 & 3,28 & 0,76 & 320 & 3,29 & 0,76 & 665 & $-0,019$ \\
\hline IMdesar7 & 3,28 & 0,83 & 357 & 3,28 & 0,80 & 323 & 3,28 & 0,82 & 680 & 0,002 \\
\hline CEinter8 & 3,11 & 0,94 & 356 & 3,16 & 0,88 & 322 & 3,13 & 0,91 & 678 & 0,050 \\
\hline CEfácil9 & 2,76 & 0,93 & 352 & 2,74 & 0,86 & 320 & 2,75 & 0,89 & 672 & $-0,023$ \\
\hline CEtrab10 & 2,79 & 1,04 & 351 & 2,78 & 0,98 & 319 & 2,78 & 1,01 & 670 & $-0,006$ \\
\hline CEúttr11 & 2,98 & 1,02 & 350 & 2,93 & 1,02 & 321 & 2,96 & 1,02 & 671 & $-0,057$ \\
\hline CEgust12 & 2,71 & 1,09 & 358 & 2,47 & 1,01 & 319 & 2,60 & 1,06 & 677 & $-0,232$ \\
\hline CEtodo13 & 3,05 & 0,99 & 353 & 3,09 & 0,93 & 323 & 3,07 & 0,96 & 676 & 0,037 \\
\hline CEútvi14 & 2,87 & 0,93 & 350 & 2,80 & 0,91 & 321 & 2,84 & 0,92 & 671 & $-0,080$ \\
\hline CEopor15 & 2,94 & 0,97 & 342 & 2,99 & 0,96 & 303 & 2,97 & 0,96 & 645 & 0,051 \\
\hline CEcrít16 & 2,53 & 1,02 & 348 & 2,52 & 0,95 & 307 & 2,52 & 0,98 & 655 & $-0,005$ \\
\hline CEcuri17 & 3,11 & 0,92 & 348 & 3,12 & 0,90 & 317 & 3,11 & 0,91 & 665 & 0,018 \\
\hline CEsalu18 & 3,00 & 0,95 & 355 & 2,87 & 0,93 & 318 & 2,94 & 0,94 & 673 & $-0,134$ \\
\hline CEimpo19 & 3,07 & 0,82 & 355 & 3,08 & 0,84 & 312 & 3,07 & 0,83 & 667 & 0,011 \\
\hline CEnatu20 & 3,03 & 0,95 & 356 & 3,02 & 0,91 & 323 & 3,02 & 0,93 & 679 & $-0,017$ \\
\hline MAreso21 & 3,05 & 0,90 & 353 & 2,98 & 0,88 & 318 & 3,02 & 0,89 & 671 & $-0,082$ \\
\hline MAcont22 & 3,47 & 0,81 & 353 & 3,57 & 0,65 & 313 & 3,52 & 0,74 & 666 & 0,130 \\
\hline CEcfco23 & 2,42 & 1,15 & 350 & 2,26 & 1,14 & 320 & 2,34 & 1,15 & 670 & $-0,138$ \\
\hline CEtrtg24 & 2,88 & 1,07 & 353 & 2,27 & 1,09 & 320 & 2,59 & 1,12 & 673 & $-0,566$ \\
\hline
\end{tabular}

Tamaño del efecto: diferencias mujeres - hombres medidas en unidades de desviación estándar.

Para elucidar la hipótesis planteada sobre la evolución con el tiempo de las actitudes de chicos y chicas, se ha realizado un análisis MANOVA global comparando las diferencias entre las dos etapas (primaria y secundaria), y también, más minuciosamente, las diferencias entre los seis grupos de grados escolares de estas etapas. En ambos casos se obtiene el mismo patrón de diferencias: las únicas variables que alcanzan diferencias estadísticamente significativas e importantes a través de las etapas y los grados son el conjunto de cuestiones de ciencia escolar $(\mathrm{F}(5,576)>9 ; \mathrm{p}<.0000000)$ y el sentido es siempre favorable a los alumnos más jóvenes. La cuestión de medio ambiente (Todos podemos hacer contribuciones importantes a la protección del medio ambiente) también presenta diferencias estadísticamente significativas $(\mathrm{F}(5,561)=3.26 ; \mathrm{p}=.007)$ favorables también a los estudiantes más jóvenes, pero su tamaño de las diferencias no es importante.

Repitiendo este mismo análisis global MANOVA separadamente para los chicos y las chicas, los resultados confirman en líneas generales el resultado anterior (tablas 3 y 4), a saber, las variables que alcanzan diferencias entre los grados estadísticamente significativas y de tamaño grande son las cuestiones referidas a la ciencia escolar, mientras las dos cuestiones de medio ambiente y las siete cuestiones de imagen de la ciencia no tienen diferencias estadísticamente significativas entre los grados. Así pues, como las variables actitudinales acerca de la ciencia escolar son las que marcan las diferencias estadísticamente significativas, el análisis subsiguiente se centra en profundizar el análisis de la evolución en cada una de esas variables. 
El descenso de las actitudes hacia la ciencia ...

Tabla 3. Puntuaciones medias de los chicos por grados de primaria $(4,5$ y 6$)$ y secundaria $(7,8$ y 9$)$.

\begin{tabular}{|c|c|c|c|c|c|c|c|}
\hline \multirow{3}{*}{ Cuestión } & \multirow{3}{*}{ Grupo } & \multicolumn{6}{|c|}{ Grados } \\
\hline & & \multicolumn{3}{|c|}{ Primaria } & \multicolumn{3}{|c|}{ Secundaria } \\
\hline & & 4 & 5 & 6 & 7 & 8 & 9 \\
\hline IMcurar1 & & 3,60 & 3,47 & 3,73 & 3,36 & 3,35 & 3,36 \\
\hline IMoportu2 & & 3,44 & 3,49 & 3,55 & 3,42 & 3,35 & 3,33 \\
\hline IMvida3 & & 3,35 & 3,21 & 3,73 & 3,22 & 3,16 & 3,22 \\
\hline IMtrabaj4 & & 3,23 & 3,07 & 3,11 & 3,06 & 2,84 & 2,84 \\
\hline IMbalan5 & & 2,91 & 3,04 & 2,80 & 2,81 & 2,63 & 2,80 \\
\hline IMimpor6 & & 3,12 & 3,54 & 3,45 & 3,33 & 3,13 & 3,19 \\
\hline IMdesar7 & & 3,17 & 3,29 & 3,18 & 3,33 & 3,28 & 3,20 \\
\hline CEinter8 & Básicas & 3,77 & 3,29 & 2,82 & 3,48 & 2,67 & 2,64 \\
\hline CEfácil9 & Básicas & 3,40 & 2,95 & 3,17 & 2,72 & 2,30 & 2,61 \\
\hline CEtrab10 & Vocacional & 3,39 & 3,34 & 2,82 & 2,98 & 2,37 & 2,31 \\
\hline CEúttr11 & Vocacional & 3,83 & 3,20 & 2,75 & 3,10 & 2,64 & 2,53 \\
\hline CEgust12 & Básicas & 3,40 & 2,83 & 2,50 & 2,55 & 2,28 & 2,53 \\
\hline CEtodo13 & Básicas & 3,47 & 3,59 & 3,27 & 3,32 & 2,77 & 2,67 \\
\hline CEútvi14 & Objetivos & 3,38 & 3,39 & 2,82 & 2,98 & 2,58 & 2,41 \\
\hline CEopor15 & Vocacional & 3,26 & 3,29 & 3,45 & 2,94 & 2,67 & 2,62 \\
\hline CEcrít16 & Objetivos & 3,02 & 2,81 & 2,25 & 2,48 & 2,27 & 2,16 \\
\hline CEcuri17 & Objetivos & 3,64 & 3,44 & 3,25 & 3,42 & 2,71 & 2,64 \\
\hline CEsalu18 & Objetivos & 3,65 & 3,57 & 3,40 & 2,86 & 2,73 & 2,64 \\
\hline CEimpo19 & Objetivos & 3,43 & 3,42 & 3,08 & 3,29 & 2,78 & 2,76 \\
\hline CEnatu20 & Objetivos & 3,60 & 3,66 & 3,27 & 3,14 & 2,61 & 2,61 \\
\hline MAreso21 & & 3,02 & 3,33 & 2,33 & 2,96 & 3,04 & 2,96 \\
\hline MAcont22 & & 3,67 & 3,59 & 3,58 & 3,49 & 3,32 & 3,33 \\
\hline CEcfco23 & Vocacional & 2,74 & 2,76 & 2,92 & 2,40 & 2,08 & 2,15 \\
\hline CEtrtg24 & Vocacional & 3,58 & 3,10 & 2,75 & 2,55 & 2,68 & 2,68 \\
\hline
\end{tabular}

La variación de las variables de actitudes hacia la ciencia escolar a través de los grados muestra, como tendencia general, que el perfil de descenso es común a todas ellas, pues a medida que aumenta el grado escolar, desde los grados iniciales hasta los finales, las puntuaciones actitudinales disminuyen. Pero también se puede constatar, que cada una de las variables muestra algunas características propias y diferenciales respecto a los demás, de modo que los perfiles de descenso particulares se podrían describir de acuerdo con las categorías básicas expuestas en el procedimiento, a saber, descenso regular, descenso abrupto en primaria, descenso abrupto en secundaria y descenso irregular. El análisis individualizado se sistematiza, sucesivamente, según los grupos de variables básicas, vocacionales y de objetivos definidos para el conjunto de variables de actitudes hacia la ciencia escolar.

\section{Actitudes básicas}

Todas las actitudes básicas (interés, facilidad, gusto y deber de estudiar más ciencia), presentan como característica propia algunos repuntes en los dos grados (6 y 7) que limitan las etapas de primaria (último) y secundaria (primero). El interés hacia la ciencia escolar (CEinter8) presenta el descenso más abrupto en los primeros grados (primaria), especialmente para los chicos, aunque estos muestran un repunte intenso en el primer curso de secundaria; el descenso de las chicas es cuantitativamente similar al de los chicos, pero más constante y 
Vázques-Alonso, Â.; Manassero-Mas M. A.

gradual, y curiosamente, el interés de las chicas es siempre superior al de sus homólogos varones en todos los grados excepto en el grado del repunte, aunque las diferencias entre chicos y chicas sólo son estadísticamente significativas en los dos grados del límite entre etapas (6 y 7$)$.

Tabla 4. Puntuaciones medias de las chicas por grados de primaria $(4,5$ y 6$)$ y secundaria $(7,8$ y 9$)$.

\begin{tabular}{|c|c|c|c|c|c|c|c|}
\hline \multirow{3}{*}{ Cuestión } & \multirow{3}{*}{ Grupo } & \multicolumn{6}{|c|}{ Grados } \\
\hline & & \multicolumn{3}{|c|}{ Primaria } & \multicolumn{3}{|c|}{ Secundaria } \\
\hline & & 4 & 5 & 6 & 7 & 8 & 9 \\
\hline IMcurar1 & & 3,22 & 3,20 & 2,94 & 3,42 & 3,25 & 3,34 \\
\hline IMoportu2 & & 3,23 & 3,36 & 3,81 & 3,15 & 3,28 & 3,45 \\
\hline IMvida3 & & 3,29 & 3,09 & 3,19 & 3,19 & 3,22 & 3,13 \\
\hline IMtrabaj4 & & 2,95 & 2,86 & 3,44 & 2,87 & 3,14 & 2,89 \\
\hline IMbalan5 & & 2,98 & 2,74 & 2,43 & 2,78 & 2,88 & 2,48 \\
\hline IMimpor6 & & 3,25 & 3,40 & 3,25 & 3,26 & 3,14 & 3,25 \\
\hline IMdesar7 & & 3,22 & 3,36 & 3,00 & 3,09 & 3,31 & 3,26 \\
\hline CEinter8 & Básicas & 3,85 & 3,33 & 3,19 & 3,11 & 2,85 & 2,73 \\
\hline CEfácil9 & Básicas & 3,20 & 2,88 & 2,69 & 2,87 & 2,59 & 2,32 \\
\hline CEtrab10 & Vocacional & 3,73 & 3,31 & 2,63 & 2,53 & 2,38 & 2,45 \\
\hline CEúttr11 & Vocacional & 3,71 & 3,02 & 3,00 & 2,89 & 2,77 & 2,62 \\
\hline CEgust12 & Básicas & 2,85 & 2,37 & 2,80 & 2,32 & 2,26 & 2,19 \\
\hline CEtodo13 & Básicas & 3,68 & 3,51 & 2,88 & 3,02 & 2,92 & 2,79 \\
\hline CEútvi14 & Objetivos & 3,68 & 2,93 & 2,53 & 2,59 & 2,57 & 2,63 \\
\hline CEopor15 & Vocacional & 3,67 & 3,37 & 2,94 & 2,89 & 2,78 & 2,77 \\
\hline CEcrít16 & Objetivos & 3,22 & 2,78 & 2,14 & 2,34 & 2,14 & 2,27 \\
\hline CEcuri17 & Objetivos & 3,71 & 3,38 & 2,67 & 3,28 & 2,69 & 2,92 \\
\hline CEsalu18 & Objetivos & 3,66 & 3,31 & 3,13 & 2,72 & 2,36 & 2,67 \\
\hline CEimpo19 & Objetivos & 3,76 & 3,07 & 3,06 & 3,06 & 2,77 & 2,85 \\
\hline CEnatu20 & Objetivos & 3,78 & 3,16 & 3,06 & 3,05 & 2,69 & 2,55 \\
\hline MAreso21 & & 2,98 & 2,86 & 3,00 & 3,19 & 2,85 & 2,94 \\
\hline MAcont22 & & 3,85 & 3,55 & 3,29 & 3,56 & 3,52 & 3,42 \\
\hline CEcfco23 & Vocacional & 2,87 & 2,62 & 2,50 & 2,19 & 1,82 & 1,69 \\
\hline CEtrtg24 & Vocacional & 2,83 & 2,80 & 2,75 & 2,30 & 1,95 & 1,83 \\
\hline
\end{tabular}

La percepción de la facilidad del aprendizaje de la ciencia escolar (CEfácil9) también muestra un perfil descendente con repuntes en todos. Los chicos presentan un descenso más irregular con sendos repuntes en los dos grados finales de etapas (6 y 9); el descenso de las chicas es más gradual, aunque también exhiben repunte en el grado 7. Los chicos perciben la ciencia más fácil que las chicas en primaria y más difícil en secundaria (excepción: el grado 9 por el repunte citado).

El descenso en la preferencia por la ciencia escolar en relación a otras asignaturas (CEgust12) es más abrupto en los chicos (el mayor descenso ocurre en primaria), mientras que parece más suave en las chicas (excepto un repunte en el grado 6) y en los grados de secundaria para los chicos. La ciencia gusta más a los chicos que a las chicas en casi todos los grados, con la excepción del grado 6 donde aparece el repunte de las chicas.

La creencia que todos deberían estudiar más ciencia en la escuela (CEtodo13) muestra un perfil de descenso abrupto un poco irregular, pero diferente en chicos y chicas. Los 
chicos puntúan más alto en tres grados y las chicas en los otros tres. Para las chicas, desciende abruptamente a lo largo de primaria y ya se mantiene bajo y estable en secundaria, mientras que para los chicos el descenso más abrupto sucede en secundaria, aunque los descensos importantes se producen después de los grados impares.

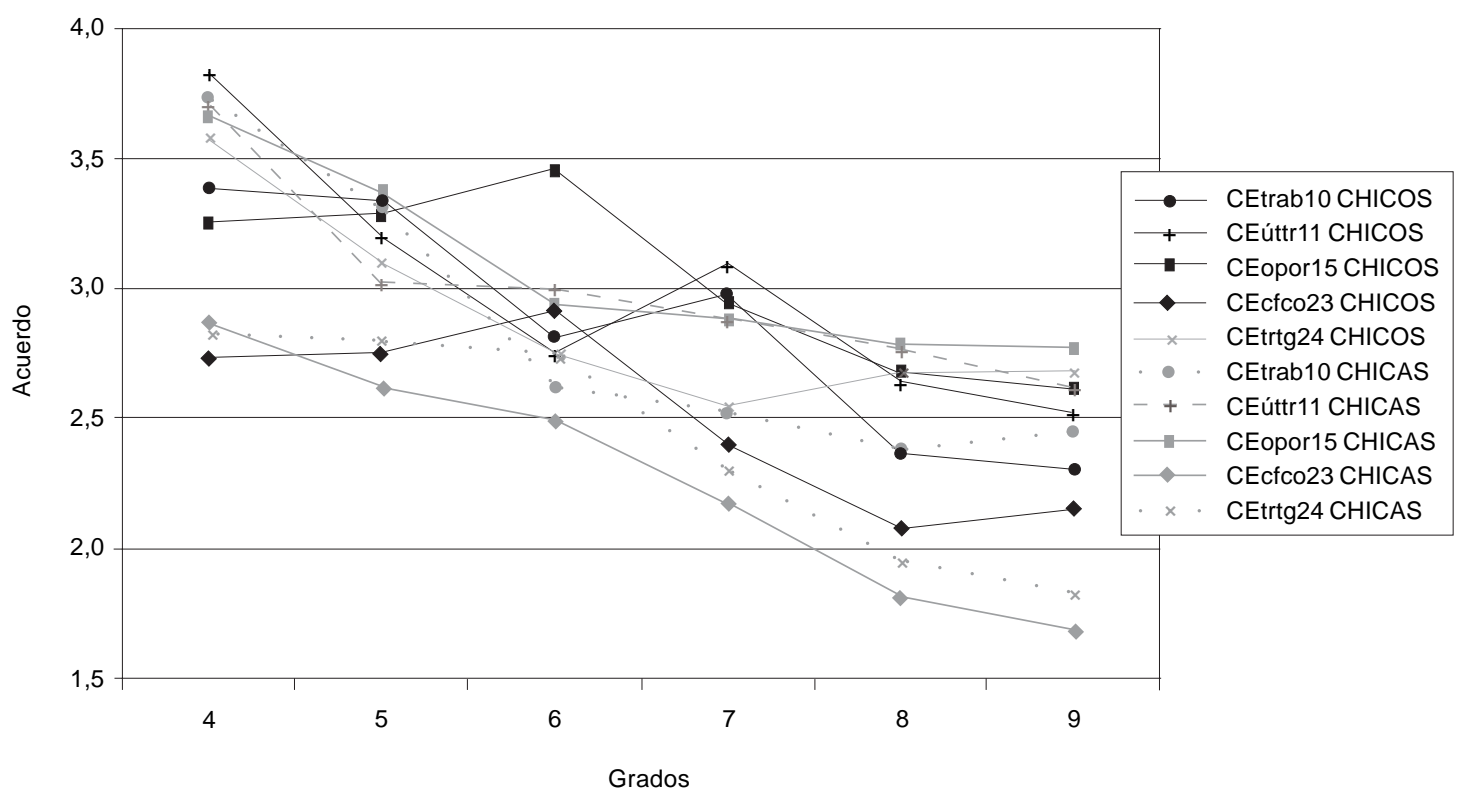

Figura 1. Perfiles evolutivos en las variables de actitudes vocacionales (cada variable se representa por el mismo tipo de línea) para los chicos (líneas más gruesas y oscuras) y las chicas (líneas más finas y claras) según los grados.

\section{Actitudes vocacionales}

Las variables actitudinales que recogen una inclinación vocacional hacia la CyT (apertura hacia trabajos interesantes - CEtrab10 -, útil para el trabajo - CEúttr11 -, mejora de las oportunidades de carrera - CEopor15 -, deseo de ser científico - CEcfco23 - y tecnólogo CEtrtg24 -) presentan perfiles un poco más diferenciados entre chicas y chicos, aunque manteniendo la tendencia general descendente con el grado. Las chicas presentan un perfil de descenso más regular que los chicos, los cuales presentan repuntes dentro de la tendencia general de descenso en estas variables. En las tres primeras variables las puntuaciones de chicas y chicos se alternan y entrelazan frecuentemente: en unos grados las chicas puntúan más alto que los chicos, y en otros grados sucede lo contrario. En cambio, en las dos últimas (deseo de ser científico o tecnólogo) los chicos tienen puntuaciones siempre superiores y aquí puede estar la raíz de la mayor huida de las mujeres de los estudios de CyT (la figura 1 representa gráficamente los perfiles de las actitudes vocacionales de chicos y chicas por grados). 
Un mismo perfil de descenso abrupto en primaria y una estabilidad relativa en secundaria se repite en varias variables vocacionales, como la utilidad para la vida cotidiana, la mejora de oportunidades de carrera en las chicas, y para la utilidad para un trabajo futuro de chicos y chicas.

La percepción de la ciencia escolar como una apertura hacia nuevos y excitantes trabajos muestra un perfil de descenso regular en los chicos y un perfil de descenso abrupto en primaria para las chicas. La percepción de la ciencia escolar como útil para un trabajo futuro muestra un perfil de descenso abrupto en primaria para las chicas y los chicos. La percepción de la ciencia escolar como mejora de las oportunidades de carrera muestra un perfil de descenso abrupto en primaria para las chicas y en secundaria para los chicos. El deseo de ser científicos muestra un perfil de descenso abrupto en secundaria para los chicos, mientras las chicas, en este caso, presentan un descenso regular. El deseo de ser tecnólogos muestra perfil de descenso abrupto para chicos y chicas pero en diferentes momentos para los chicos (en primaria) que para las chicas (en secundaria).

\section{Actitudes hacia los objetivos de la ciencia}

Las variables que recogen la actitud de los estudiantes hacia los objetivos de la ciencia escolar (aumentar la curiosidad - CEcuri17 -, espíritu crítico - CEcrít16 -, utilidad CEútvi14 -, importancia - CEimpo19 -, cuidar la salud - CEsalu18 - y aprecio por la naturaleza - CEnatu20 -) muestran también el perfil general de descenso actitudinal bastante regular, con excepción de la curiosidad que exhibe un perfil de descenso con amplios dientes de sierra y con las puntuaciones de chicos y chicas bastante próximas en general. También se observan especificidades como que la utilidad para la vida cotidiana en las chicas desciende abruptamente en primaria y se mantiene estable en secundaria, y este mismo perfil se repite en el caso del espíritu crítico de chicos y chicas.

La percepción de la utilidad de la ciencia escolar para la vida diaria muestra un perfil de descenso regular en los chicos y un perfil de descenso abrupto en primaria para las chicas. La percepción de la ciencia escolar como formadora del espíritu crítico y escéptico muestra un perfil de descenso abrupto en primaria para las chicas y los chicos. La percepción de la ciencia escolar como enseñanza para cuidar la salud muestra un perfil de descenso apreciablemente regular para las chicas y los chicos. La percepción de la ciencia escolar como cultura de la importancia de la ciencia muestra un perfil de descenso abrupto en primaria para las chicas y un poco más irregular para los chicos. La percepción de la ciencia escolar como enseñanza para apreciar la naturaleza muestra un perfil de descenso aproximadamente regular para las chicas y los chicos.

Finalmente, el balance del descenso global de las actitudes no es sustancialmente diferente para chicos y chicas. Un parámetro sensible a todo el proceso global se puede construir considerando todos los descensos en cada cuestión individual para chicos y chicas (sumando las diferencias obtenidas entre el primer grado y el último en todas las cuestiones); la magnitud obtenida para este indicador global del declive es similar para chicos y chicas. No obstante, aparecen diferencias en algunas cuestiones donde las chicas disminuyen relativamente más que los chicos (balance entre beneficios y perjuicios, oportunidades de carrera, importancia, aprecio por la naturaleza y deseo de ser científico) y otras donde los chicos bajan más 
que las chicas (curar enfermedades, oportunidades futuras, nuevos trabajos, útil para un trabajo y gusto por la ciencia escolar).

En suma, se puede concluir, pues, que la magnitud del declive actitudinal es similar para los chicos y para las chicas, aunque las diferencias en algunas cuestiones y en la evolución de estas diferencias a lo largo de los seis grados muestran rasgos diferentes entre ambos grupos.

\section{Discusión}

Este estudio extiende y confirma con estudiantes españoles de primaria y secundaria una validación empírica de la hipótesis del descenso de las actitudes hacia la ciencia que tiene lugar desde la infancia hasta la adolescencia con una perspectiva de género y compara los perfiles diferenciados que exhiben hombres y mujeres en este descenso. Para verificar esta hipótesis se toma como unidad de análisis temporal el grado escolar o curso y como criterios de verificación de la hipótesis el cumplimiento simultáneo de dos condiciones: la existencia de diferencias estadísticamente significativas entre los grados y un perfil de descenso a lo largo de los grados sucesivos.

La primera conclusión del estudio es que no todas las variables de actitudes hacia la CyT presentan este declive. En particular, las actitudes relativas a la imagen de la CyT y las actitudes hacia el medio ambiente no presentan este declive evolutivo, en la mayoría de los casos porque no cumplen la primera de las condiciones.

La segunda conclusión es que las actitudes referidas a la ciencia escolar verifican la hipótesis del declive de las actitudes de los estudiantes con el grado escolar, porque estas variables cumplen simultáneamente las dos condiciones impuestas, mostrar diferencias significativas entre los grados y un perfil de descenso a lo largo de los seis grados estudiados.

La tercera conclusión se refiere a las diferencias de actitudes entre chicos y chicas. Aunque los chicos tienen actitudes generalmente más positivas que las chicas en la mayoría de las actitudes evaluadas, las diferencias globales entre chicos y chicas son bajas, porque no se observan diferencias estadísticamente significativas en las puntuaciones actitudinales. Sólo dos cuestiones exhiben diferencias estadísticamente significativas favorables a los chicos con un tamaño del efecto moderado y grande, a saber, el gusto por la ciencia escolar (en relación a las demás asignaturas) y el deseo de tener un trabajo relacionado con la tecnología, que exhibe las diferencias más relevantes entre chicos y chicas adolescentes.

La cuarta conclusión se refiere a las diferencias en los perfiles de evolución de chicos y chicas en las variables de la ciencia escolar, que tienen una implicación directa para la educación científica porque están relacionados diferencialmente con las etapas educativas (primaria y secundaria) y que se resumen a continuación.

La primera característica a resaltar es que chicos y chicas comparten el patrón general de descenso de las puntuaciones, es decir, chicos y chicas empeoran sus actitudes relacionadas con la ciencia escolar en todas las variables actitudinales hacia la ciencia escolar. Chicos y chicas tienen puntuaciones actitudinales más altas cuando son más jóvenes que cuando se hacen mayores, sobre un período de seis años que corresponde a edades aproximadas entre 10 y 15 años, y esto sucede sin excepción en todas las variables actitudinales relativas a la ciencia escolar. 
La segunda característica es que la evolución de este descenso a lo largo de los seis años no es siempre regular, sino que en muchas ocasiones la mayor proporción de este descenso sucede en una de las dos etapas del período (descenso abrupto) en la inicial (primaria) o bien la final (secundaria), de modo que las actitudes en la otra etapa complementaria permanecen prácticamente constantes: si el descenso abrupto ocurre en primaria, apenas varían en secundaria, y viceversa.

Las diferencias en la evolución de las actitudes de chicos y chicas se centran en los diferentes perfiles de descenso actitudinal de las variables. El perfil de descenso aproximadamente regular a lo largo de todos los grados se observa en chicos y chicas para las variables actitudinales siguientes: cuidar la salud, fácil de aprender, importancia de la ciencia y aprecio de la naturaleza; también sólo para el caso de los chicos, en la apertura a nuevos trabajos, utilidad para la vida cotidiana y curiosidad, y sólo en el caso de las chicas, el interés y el deseo de ser científicos.

El perfil de decremento actitudinal abrupto en los primeros grados (primaria) es común para chicos y chicas en las variables gusto por la asignatura de ciencia, utilidad para el trabajo futuro y ser críticos y escépticos. Diferencialmente para chicos y chicas, también se distingue este perfil, en el interés y el deseo de un trabajo en tecnología (sólo para chicos), y en la apertura hacia nuevos y excitantes trabajos, la utilidad para la vida cotidiana, la creencia que todos deberían estudiar más ciencia en la escuela, la curiosidad y el aumento de oportunidades de carrera (sólo para las chicas).

El perfil decremento actitudinal abrupto en los últimos grados (secundaria) es el menos frecuente, y sólo se observa diferencialmente entre chicos y chicas; en el caso de la creencia en que todos deberían estudiar más ciencia en la escuela, la mejora de las oportunidades de carrera y el deseo de ser científico (sólo en chicos) y el deseo de un trabajo en tecnología (sólo en chicas).

En suma, este estudio confirma el patrón de descenso actitudinal hallado en otros estudios examinados en la introducción, y aunque algunas actitudes decrecen regularmente a lo largo de los diferentes grados, otras concentran su descenso más abruptamente en los primeros o últimos grados. Respecto a la controversia sobre si este descenso tiene lugar en el final de la etapa de primaria (PELL; JARVIS, 2001; MURPHY; BEGGS, 2003) o más bien en secundaria (OSBORNE; DRIVER; SIMON, 1998; PARKINSON et al., 1998; RAMSDEN, 1998; SIMPSON; OLIVER, 1990; WEINBURG, 1995), las evidencias encontradas aquí permiten precisar que depende del género (chicos o chicas) y de la variable actitudinal considerada. El perfil aproximadamente regular y constante es más común en los chicos, mientras el perfil de descenso concentrado en primaria aparece más entre las chicas, las cuales, curiosamente, también superan a los chicos en el primer grado en muchas variables. Los chicos tienden a presentar perfiles cuyo descenso abrupto sucede en secundaria más que las chicas. Sin duda, se requieren más investigaciones que permitan corroborar los detalles de variaciones diferenciales según la etapa, que, en muchos casos aparecen ligados al sexo, como factores diferenciales entre chicos y chicas.

Los hallazgos de este estudio tienen implicaciones para la investigación y para la educación científica. La investigación ha sugerido el declive de las actitudes hacia la ciencia entre los jóvenes adolescentes con la edad, pero este estudio añade matices importantes para la investigación futura. El declive actitudinal no ocurre en todas las variables, sino que depende 
del objeto actitudinal específico: el declive afecta principalmente a las actitudes englobadas bajo la denominación de actitudes hacia la ciencia escolar, y con más intensidad a los objetos denominados las actitudes básicas (interés, facilidad, gusto, etc.). Además, aparece ligado al sexo de los estudiantes.

Las consecuencias de los resultados expuestos para la educación científica parecen más importantes. En primer lugar, este estudio identifica un punto muy débil de la educación científica: enseñar ciencia en la escuela durante años para que los alumnos deterioren sus actitudes hacia la ciencia de una manera tan evidente es un resultado tan injustificable como inadmisible desde cualquier punto de vista. En segundo lugar, reitera un mensaje de alarma: el significativo declive de las actitudes de los estudiantes hacia la ciencia escolar a lo largo de primaria y secundaria que muestra este estudio (y otros ya citados), pone en valor que el primero de los objetivos de la educación en ciencias debe ser evitar a toda costa este deterioro actitudinal, como clama FENSHAM (2004) y recomienda el informe Rocard (ROCARD, 2007).

Ante este panorama, la cuestión a plantear a la educación científica es, a la vez, simple y demoledora ¿es aceptable e incluso rentable seguir enseñando y aprendiendo ciencias para que después de seis años los estudiantes presenten unas actitudes hacia la ciencia escolar tan deterioradas respecto al entusiasmo inicial? Las consideraciones anteriores sugieren dos reflexiones inmediatas: primero, conseguir actitudes positivas hacia la ciencia no parece que sea un objetivo real de la práctica actual de la enseñanza de la ciencia; segundo, incluso en el caso que este fuera un objetivo real e importante de la enseñanza de la ciencia escolar, parece evidente que la práctica educativa actual no consigue que los estudiantes mantengan sus positivas actitudes iniciales, o incluso las mejoren. En cualquiera de los casos, la educación científica escolar debería plantearse con seriedad la educación de las actitudes relacionadas con la ciencia, lo cual implica planificarlas en el currículo, trabajarlas en el aula y, finalmente, evaluarlas, en el mismo nivel que los demás contenidos conceptuales (AIKENHEAD, 2003; VÁZQUEZ ALONSO; ACEVEDO-DÍAZ; MANASSERO MAS, 2005; VÁZQUEZ ALONSO; MANASSERO MAS, 2007b, 2007c), como ha decidido ya el programa PISA (ORGANISATION FOR ECONOMIC CO-OPERATION AND DEVELOPMENT, 2007).

El creciente rechazo de la ciencia por chicos y chicas adolescentes sugiere dos interesantes consideraciones estratégicas finales para la educación. En las últimas décadas, la investigación sobre concepciones alternativas (ver por ejemplo, POZO; GOMEZ, 1998) ha reiterado que los estudiantes fracasan en aprender significativamente los contenidos cognitivos y conceptuales de ciencias. Los resultados de este estudio sobre el declive actitudinal podrían estar señalando, a la vez, una causa posible de ese fracaso cognitivo y una posible solución al dilema de la dificultad del aprendizaje de la ciencia y el cambio conceptual. Los estudiantes no aprenden porque los factores actitudinales y afectivos, que regulan los aprendizajes generales y fundamentan la motivación de aprender, son crecientemente negativos con la edad para más estudiantes, cuyas posibilidades de aprendizaje disminuyen progresivamente, en lugar de mejorarse. Por otro lado, en la mayoría de los países el período final de la educación obligatoria es crucial en la toma de decisiones sobre elección de estudios y, en particular, para el inicio de una vocación científica; la coincidencia en este período del declive actitudinal hacia la ciencia mostrado en este estudio y la toma de decisiones vocacionales, especialmente acerca de la intención de ser científicos o trabajar en tecnología, hace que estas decisiones se tomen en un contexto muy adverso (creciente desinterés y actitudes negativas), de modo que no resulta 
Vázques-Alonso, Â.; Manassero-Mas M. A.

extraño el rechazo de la ciencia como carrera y profesión, que seguramente causa la falta de vocaciones científicas que preocupa especialmente en muchos países y en la Unión Europea (CONSEJO DE EUROPA, 2003; GAGO, 2004; ROCARD, 2007).

Estos argumentos sugieren que la mejora de las actitudes de los estudiantes debería ser un objetivo de capital importancia como instrumento de motivación de los jóvenes estudiantes, para mejorar su aprendizaje de la ciencia y su percepción más adecuada de las diferentes opciones de carrera, para el fomento de las vocaciones científicas (VÁZQUEZ ALONSO; MANASSERO MAS, 2010).

\section{Referencias}

AIKENHEAD, G. S. Review of research on humanistic perspectives in science curricula. In: CONFERENCE OF THE EUROPEAN SCIENCE EDUCATION RESEARCH ASSOCIATION, 4., 2003, Noordwijkerhout. Comunications... Noordwijkerhout, 2003. Disponible en: <http://www.usask.ca/education/people/aikenhead/ESERA_2.pdf.>. Accesado en: 18 mayo 2004.

AJZEN, I.; FISHBEIN, M. Understanding attitudes and predicting social behavior. Englewood Cliffs: Prentice Hall, 1980.

BAIRD, J. R.; PENNA, C. Perceptions of challenge in science learning. International Journal of Science Education, s.l., v. 19, n. 10, p. 1195-1209, 1997.

BREAKWELL, G. M.; BEARDSELL, S. Gender, parental and peer influences upon science attitudes and activities. Public Understanding of Science, s.l., v. 1, n. 2, p. 183-197, 1992.

CONSEJO DE EUROPA. Conclusiones del Consejo de 5 de mayo de 2003 sobre los niveles de referencia del rendimiento medio europeo en educación y formación. Diario Oficial C, n. 134, 7 jun. 2003. Disponible en: <http://europa.eu.int/scadplus/leg/es/cha/ c11064.htm>. Accesado en: 10 ene. 2006.

EAGLY, A. H.; CHAIKEN, S. The psychology of attitudes. Forth Worth: Harcourt Brace College Publishers, 1993.

FAZIO, R. H. How do attitudes guide behavior? In: SORRENTINO, R. M.; HIGGINS, E. T. (Ed.). Handbook of motivation and cognition: foundations of social behavior. New York: Guilford Press, 1986. p. 3-19.

FENSHAM, P. J. Beyond knowledge: other scientific qualities as outcomes for school science education. In: JANIUK, R. M.; SAMONEK-MICIUK, E. (Ed.). Science and technology education for a diverse world: dilemmas, needs and partnerships. Lublin (Polonia): Maria Curie-Sklodowska University Press, 2004. p. 23-25.

FERGUSON, P. D.; FRASER, B. J. The role of school size and gender in students' perceptions of science during the transition from elementary to high school. In: ANNUAL MEETING OF THE NATIONAL ASSOCIATION FOR RESEARCH IN SCIENCE TEACHING, 1996, St. Louis. Paper... St. Louis: MO, 1996. p. 1-20. 
El descenso de las actitudes hacia la ciencia $\ldots$

GAGO, J. M. Europe needs more scientists. Brussels: European Community Conference Increasing Human Resources for Science and Technology, 2004. Disponible en: <http:// europa.eu.int/comm/research/conferences/2004/sciprof/publications_en.html>. Accesado en: 7 nov. 2005.

GARDNER, P. L. Attitudes to science: a review. Studies in Science Education, s.l., v. 2, n. 1, p. 1-41, 1975.

GEORGE, R. Measuring change in students' attitudes toward science over time: an application of latent variable growth modeling. Journal of Science Education and Technology, s.l., v. 9, n. 3, p. 213-225, 2000.

A cross domain analysis of change in students' attitudes toward science and attitudes about the utility of science. International Journal of Science Education, s.l., v. 28, n. 6, p. 571-589, 2006.

GIBSON, H. L.; CHASE, C. Longitudinal impact of an inquiry-based science program on middle school students' sttitudes toward science. Science Education, s.l., v. 86, n. 5, p. 693-705, 2002.

GREENFIELD, T. A. Gender ethnicity, science achievement, and attitudes. Journal of Research in Science Teaching, s.l., v. 33, n. 8, p. 901-933, 1996.

HAVARD, N. Student attitudes to studying A-level sciences. Public Understanding of Science, s.l., v. 5, n. 4, p. 321-330, 1996.

HENDLEY, D. et al. Gender differences in pupil attitudes to the national curriculum foundation subjects of English, mathematics, science and technology in Key Stage 3 in South Wales. Educational Studies, s.l., v. 21, n. 1, p. 85-97, 1995.

HENDLEY, D.; STABLES, S.; STABLES, A. Pupils' subject preferences at Key Stage 3 in South Wales. Educational Studies, s.l., v. 22, n. 2, p. 177-186, 1996.

INSTITUTO NACIONAL DE EVALUACIÓN Y CALIDAD DEL SISTEMA EDUCATIVO. Evaluación de la educación secundaria obligatoria 2000. Madrid: MECD, INECSE, 2003.

JENKINS, E. W.; NELSON, N. W. Important but not for me: students' attitudes towards secondary school science in England. Research in Science and Technological Education, s.l., v. 23, n. 1, p. 41-57, 2005.

JIMÉNEZ, M. P.; ÁLVAREZ, M. Género, ciencia y tecnología. In: MORENO, M. (Ed.). Del silencio a la palabra. Madrid: Instituto de la Mujer, 1992. p. 178-196.

LEE, V. E.; BRYK, A. S. Effects of single-sex secondary schools on student achievement and attitudes. Journal of Educational Psychology, s.l., v. 78, n. 5, p. 81-95, 1986.

MANASSERO MAS, M. A.; VÁZQUEZ-ALONSO, A. Factores determinantes de las actitudes relacionadas con la ciencia. Revista Española de Pedagogía, Madrid, v. 54, n. 203, p. 43-77, 1996. 
Vázques-Alonso, Â.; Manassero-Mas M. A.

MONGUILLOT, I. La valoración de los alumnos de la educación secundaria. In: MARCHESI, A.; MARTÍN, E. (Orgs.). Evaluación de la educación secundaria: fotografía de una etapa polémica. Madrid: Fundación Santa María, 2002. p. 273-286.

MURPHY, C.; BEGGS, J. Children perceptions of school science. School Science Review, Hatfield, v. 84, n. 308, p. 109-116, 2003.

ORGANISATION FOR ECONOMIC CO-OPERATION AND DEVELOPMENT. PISA 2006: science competencies for tomorrow's world. Brussels: OECD, 2007. v. 1.

ORMerOD, M. B.; DUCKWORTH, D. Pupils attitudes' to science: a review of research. Windsor: NFER Publishing, 1975.

OSBORNE, J.; DRIVER, R.; SIMON, S. Attitudes to science: issues and concerns. School Science Review, Hatfield, v. 79, n. 288, p. 27-33, 1998.

OSBORNE, J.; SIMON, S.; COLLINS S. Attitudes towards science: a review of the literature and its implications. International Journal of Science Education, s.1., v. 25, n. 9, p. 1049-1079, 2003.

PARKINSON, J. et al. Pupils' attitudes to science in key stage 3 of the national curriculum: a study of pupils in South Wales. Research in Science \& Technological Education, s.l., v. 16, n. 2, p. 165-176, 1998.

PELL, T.; JARVIS, T. Developing attitude to science scales for use with children of ages from five to eleven years. International Journal of Science Education, s.l., v. 23, n. 8, p. 847- 862, 2001.

PÉREZ, A. Evaluación nacional de actitudes y valores hacia la ciencia en entornos educativos. Madrid: FECYT, 2005.

PIBURN, M. D.; BAKER, D. R. If I were the teacher ... qualitative study of attitude towards science. Science Education, s.l., v. 77, n. 4, p. 393-406, 1993.

POZO, J. I.; GOMEZ, M. A. Aprender y enseñar ciencia. Madrid: Morata/MEC, 1998.

RAMSDEN, J. M. Mission impossible?: can anything be done about attitudes to science? International Journal of Science Education, s.l., v. 20, n. 2, p. 125-137, 1998.

ROCARD, M. Science education now: a renewed pedagogy for the future of Europe. Brusselss: European Commission, 2007.

SCHIBECI, R. A. Attitudes to science: un update. Studies in Science Education, s.l., v. 11, n. 1, p. 26-59, 1984.

SCHREINER, C.; SJØBERG, S. Sowing the seeds of ROSE: background, rationale, questionnaire development and datacollection for ROSE (the relevance of science education): a comparative study of students' views of science and science education. Acta Didactica, Oslo, n. 4, p. 1-120, 2004. Disponible en: < http://www.ils.uio.no/forskning/ rose/documents/AD0404.pdf>. Accesado en: 18 mayo 2009. 
El descenso de las actitudes hacia la ciencia ...

SIMPSON, R. D.; OLIVER, J. E. A summary of major influences on attitude toward an achievement in science among adolescent students. Science Education, s.l., v. 74, n. 1, p. 1-18, 1990.

SJØBERG, S. Science and scientists: the SAS-study: cross-cultural evidence and perspectives on pulpils' interests, experiences and perceptions - background, development and selected results. Oslo: University of Oslo, Department of Teacher Education and School Development, 2000.

Science education: the voice of the learners. In: CONFERENCE ON INCREASING HUMAN RESOURCES FOR SCIENCE AND TECHNOLOGY IN EUROPE, 2004, Brussels. Disponible en: <http://roseproject.no/network/countries/ norway/eng/nor-sjoberg-eu2004.pdf>. Accesado en: 8 jul. 2006.

Young people and science: attitudes, values and priorities - evidence from the ROSE project. In: EU's SCIENCE AND SOCIETY FORUM, INCREASING HUMAN RESOURCES FOR SCIENCE AND TECHNOLOGY IN EUROPE, 2005, Brussels. Disponible en: <http://www.ils.uio.no/english/rose/publications/englishpresentations.html>. Accesado en: 2 mayo 2006.

SPEERING, W.; RENNIE, L. Students' perceptions about science: the impact of transition from primary to secondary school. Research in Science Education, s.l., v. 26, n. 3, p. 283-298, 1996.

TRUMPER, R. Science and technology education for a diverse world: dilemmas, needs and partnerships. Lublin (Polonia): Maria Curie-Sklodowska University Press, 2004.

VÁZQUEZ ALONSO, A.; MANASSERO MAS, M. A. Actitudes relacionadas con la ciencia: una revisión conceptual. Enseñanza de las Ciencias, Barcelona, v. 13, n. 3, p. 337-346, 1995.

;___ L L L relevancia de la educación científica. Palma de Mallorca:

Universitat de les Illes Balears, Servei de Publicacions i Intercanvi Cientific, 2007a.

.;___ En defensa de las actitudes y emociones en la educación científica (I):

evidencias y argumentos generales. Revista Eureka sobre Enseñanza y Divulgación de las Ciencias, Cádiz, v. 4, n. 2, p. 247-271, 2007b. Disponible en: <http://www.apaceureka.org/revista/Volumen4/Numero_4_2/Vazquez_Manassero_2007.pdf $>$. Accesado en: 7 jul. 2009.

.; E___ En defensa de las actitudes y emociones en la educación científica (II): evidencias empíricas derivadas de la investigación. Revista Eureka sobre Enseñanza y Divulgación de las Ciencias, Cádiz, v. 4, n. 3, p. 417-441, 2007c. Disponible en: <http:// www.apac-eureka.org/revista/Volumen4/Numero_4_3/Vazquez_Manassero_2007b.pdf>. Accesado en: 7 jul. 2009.

;_ Patrones actitudinales de la vocación científica y tecnológica en chicas y chicos de secundaria. Revista Iberoamericana de Educación/Revista Iberoa-americana de Educação, Madrid, v. 50, n. 4, p. 4-25, 2009. 
Vázques-Alonso, Â.; Manassero-Mas M. A.

.; Perfiles actitudinales de la elección de ciencias en secundaria según el sexo y el tipo de educación. Revista Electrónica de Enseñanza de las Ciencias, Vigo, v. 9, n. 1, p. 242-260, 2010.

VÁZQUEZ ALONSO, A; ACEVEDO-DÍAZ, J. A.; MANASSERO MAS, M. A. Más allá de la enseñanza de las ciencias para científicos: hacia una educación científica humanística. Revista Electrónica de Enseñanza de las Ciencias, Vigo, v. 4, n. 2, p. 1-30, 2005. Disponible en: <http://www.saum.uvigo.es/reec/volumenes/volumen4/ ART5_Vol4_N2.pdf>. Accesado en: 4 jul. 2006.

WEINBURGH, M. Gender differences in student attitudes towards science: a metaanalysis of the literature from 1970 to 1991. Journal of Research in Science Teaching, s.l., v. 32, n. 4, p. 387-398, 1995.

Apéndice. Cuestionario de actitudes hacia la ciencia y la tecnología.

¿En qué grado estás de acuerdo con las siguientes frases referidas a la ciencia o tecnología?

IMcurar1 El progreso científico y tecnológico ayuda a curar enfermedades como SIDA, cáncer etc.

IMoportu2 Gracias a la ciencia y la tecnología habrá mejores oportunidades para las generaciones futuras

IMvida3 La ciencia y la tecnología hacen nuestra vida más saludable, más fácil y más cómoda

IMtrabaj4 La aplicación de ciencia y las nuevas tecnologías harán los trabajos más interesantes

IMbalan5 Los beneficios de la ciencia son mayores que los efectos perjudiciales que podría tener

IMimpor6 La ciencia y tecnología son importantes para la sociedad

IMdesar7 Un país necesita ciencia y tecnología para llegar a desarrollarse

CEinter8 La ciencia que he aprendido en la escuela es interesante

CEfácil9 La ciencia escolar es fácil de aprender

CEtrab10 La ciencia escolar me ha abierto los ojos a nuevos y excitantes trabajos

CEúttr11 La ciencia de la escuela me será útil en mi trabajo futuro

CEgust12 La ciencia escolar me gusta más que la mayoría de las otras asignaturas

CEtodo13 Yo creo que todos deberían aprender ciencia en la escuela

CEútvi14 Las cosas que aprendo en la ciencia escolar son útiles en mi vida cotidiana

CEopor15 Pienso que la ciencia que aprendo en la escuela mejora mis oportunidades en mi carrera

CEcrít16 La ciencia escolar me ha hecho más crítico y escéptico

CEcuri17 La ciencia escolar ha aumentado mi curiosidad sobre las cosas que todavía no se pueden explicar

CEsalu18 La ciencia escolar me ha enseñado a cuidar mi salud

CEimpo19 La ciencia escolar me ha demostrado la importancia de la ciencia para nuestra manera de vivir

CEnatu20 La ciencia escolar ha aumentado mi aprecio por la naturaleza

MAreso21 La ciencia y la tecnología pueden resolver los problemas del medio ambiente

MAcont22 Todos podemos hacer contribuciones importantes a la protección del medio ambiente

CEcfco23 Me gustaría llegar a ser un científico

CEtrtg24 Me gustaría conseguir un trabajo en tecnología

Artigo submetido em agosto de 2010 e aceito em janeiro de 2011. 